\title{
A Structure and Function Analysis of DNA
}

\author{
Monolayers Created from Self-Assembling DNA \\ Dendron Conjugates
}

Christian N. Warner, Zachary D. Hunter, Destiny D. Carte, Tyler J. Skidmore, Erik S.

Vint, and B. Scott Day*

Department of Chemistry, Marshall University, Huntington, WV 25755

DNA-Dendron Conjugate Synthesis:

\section{A. Synthesis}

Figure S1 shows a representative synthesis scheme for the conjugation of the ssDNA molecules to the polyamidoamine (PAMAM) dendrons. Enough $N$-Succinimidyl-S-acetylthiopropionate (SATP) was weighed to make a two to one ratio of SATP to primary amines on the dendrimers. 
The weighed SATP was then dissolved in $150 \mu \mathrm{L}$ of DMSO in a $1.5 \mathrm{~mL}$ conical vial and diluted to a total volume of $500 \mu \mathrm{L}$ with $100 \mathrm{mM}$ phosphate buffer at $\mathrm{pH} 7.25$. The dendrimer was then added to the solution and mixed using a vortex mixer. The resulting solution was allowed to sit for 45 minutes at room temperature in order to complete the nucleophilic substitution reaction between the primary amine on the dendrimers' periphery and the NHS ester of SATP. This step of the synthesis placed a protected thiol group as a thioacetate on the dendrimer periphery (Figure S1A). The thioacetate terminated dendrimers were then reacted with a 10:1 ratio of tris-(2-carboxyethyl) phosphine (TCEP) to dendrimer in order to cleave the disulfide bond in the dendrimer core. After the disulfide cleavage reaction, excess SATP, TCEP and other small molecule by-products were removed by centrifugation with $10 \mathrm{~K}$ MWCO Amicon microcentrifugation columns while simultaneously exchanging to a $100 \mathrm{mM}$ Phosphate buffered solution at $\mathrm{pH} 6.8$.

Amine-terminated probe strand DNA oligonucleotides were reacted with a 100 fold molar excess of linker molecules (Figure S2) in $100 \mathrm{mM}$ phosphate buffer at $\mathrm{pH} 7.25$. The primary amine on the DNA reacts with the NHS ester to form a stable amide bond by a nucleophilic substitution reaction (Figure S1C shows sulfo-SMCC as example). This reaction was incubated for 45 minutes at room temperature before purifying the product using an Amicon 10K MWCO centrifugation column and exchanging for a $\mathrm{pH} 6.8$ buffer. The purified DNA-linker product was then reacted with a 10 -fold excess of purified dendron product and allowed to incubate at room temperature overnight. The maleimido group of the linker reacts with the sulfhydryl-containing dendron to form a stable thioether bond (Figure S1D). It is important to note that the efficiency of this reaction increases with increasing concentration of both the linker-modified DNA and the dendrons. 


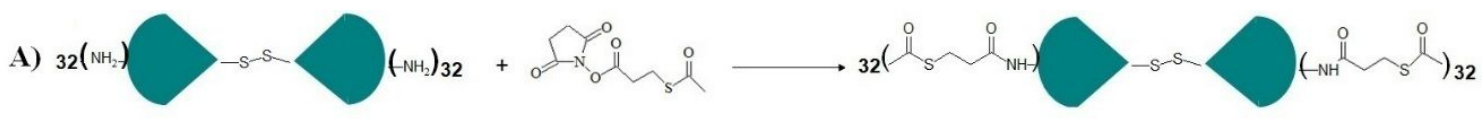

B)

C)

D)

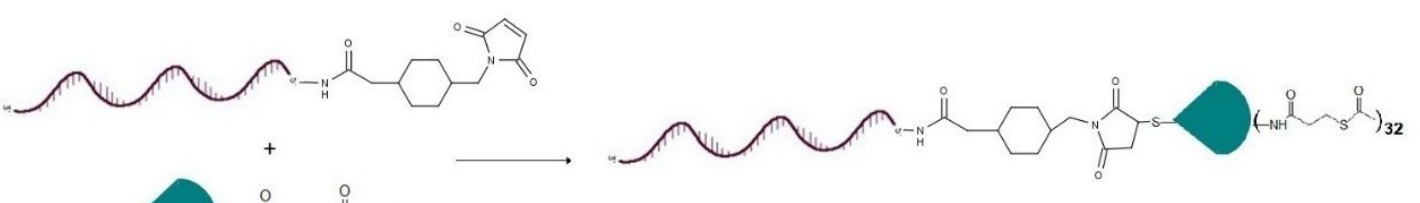

(- 2 (-

E)

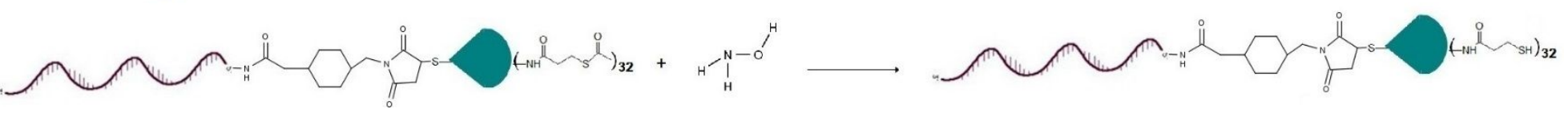

Figure S1: Synthesis scheme of DNA-dendron conjugates from a G4 cystamine core PAMAM dendrimer. (A) Functionalization of PAMAM dendrimers with SATP, (B) Reduction of disulfide dendrimer core using TCEP, (C) Addition of sulfo-SMCC to amine-terminated ssDNA, (D) Conjugation of ssDNA to thioacetate-terminated dendron, (E) Deacetylation of conjugate using hydroxylamine. 
<smiles>[R]NC(=O)CN1C(=O)CC([R7])C1=O</smiles>

AMAS (4.4 Å)<smiles>[R]SC(=O)CCCN1C(=O)CC([R7])C1=O</smiles>

$\operatorname{GMBS}(7.3 \AA ̊)$<smiles>[R]NC(=O)CCCCCCCCCCN1C(=O)CC([R17])C1=O</smiles>

KMUS (16.3 Å)<smiles>[R]NC(=O)CCCCCNC(=O)C1CCC(CN2C(=O)CC([R1])C2=O)CC1</smiles>

LC-SMCC (16.1 Å)<smiles>[R]NC(=O)C1CCC(CN2C(=O)CC([R7])C2=O)CC1</smiles>

$\operatorname{SMCC~(8.3~\AA ̊)~}$

Figure S2: Structures of linker molecules as they would appear in the DNA-dendron conjugate molecules. For all linkers, R' represents the dendron and R represents the DNA sequence. The letters are to describe the original structure names and the values in the parentheses are the "spacer arm" length given from the supplier, ThermoScientific TM.

\section{B. Surface Density Calculations}

\section{a. Calculations from SPR data}

Calibration curves from SPR angular shifts from bulk solutions were used to measure

the instrument's response to refractive index changes. Over the narrow range of angular shifts measured in these experiments, the instrumental response was linear with bulk refractive index. The slope of this line was used to convert the SPR signal measured from monolayer formation to refractive index changes at the gold surface. The refractive index 
changes were used to calculate concentrations of conjugates from refractive index increments $\left(0.14 \mathrm{~mL} / \mathrm{g}^{1}\right.$ for DNA and $0.18 \mathrm{~mL} / \mathrm{g}$ for dendrimers). The value for the refractive index increment of the dendrimers was estimated by taking a weighted average of values for the amino acids glycine, lysine and cysteine. ${ }^{2}$ Finally, the surface coverage was calculated by multiplying the concentrations by the film thicknesses.

To obtain monolayer thickness values for the conjugate molecules, the molecular components were added together and assumed to stretch perpendicular to the planar gold. The chemical supplier's information was used to estimate the height of the dendrons (from dendrimer radius) and the linker lengths. The short, alkane chain from the DNA modification was assumed to be $1.0 \mathrm{~nm}$ and the ssDNA was assumed to be two times the length of dsDNA for a 25 base pair sequence. ${ }^{1}$ As an example, the monolayer formed from the G4 DNA-dendron conjugate using the sulfo-SMCC linker was calculate to be $20.6 \mathrm{~nm}((4.4 / 2) \mathrm{nm}+0.8 \mathrm{~nm}+1.0 \mathrm{~nm}+16.6 \mathrm{~nm})$. The film thickness for the single thiol DNA monolayers only included the short alkane chain and the ssDNA. 
The instrument response to target DNA was assumed to be the same as the instrument response to the probe DNA binding for the single thiol DNA monolayers. Instrumental angular responses were converted to surface density and then used to calculate hybridization efficiencies from the ratio of target surface density to probe surface density.

\section{b. Calculations for estimated conjugate probe densities}

To estimate the probe densities for Figure 3, the area per molecule was calculated assuming each molecule occupies a square area with sides equal to the dendrimer radius. The radii used for this calculation were taken from the manufacturer's theoretical values (Andrews ChemServices) as $2.6 \mathrm{~nm}$ for generation 2, $3.6 \mathrm{~nm}$ for generation 3, 4.4 $\mathrm{nm}$ for generation 4 , and 5.7 for generation 5 .

\section{Results}

\section{a. Conjugate Purification}

Purification of the DNA-dendron conjugates was accomplished using $4 \%$ agarose gels. Traditionally, separation of small strands of DNA, such as the 25 base strand used in this study, is performed using polyacrylamide gels. However, it was difficult to remove small pieces of 
polyacrylamide from the recovered conjugates, therefore causing interference with monolayer formation.

Figure S3 (left) shows a representative gel image of generation two (G2) and four (G4) DNAdendron conjugate synthesis products along with several control lanes. The G4 conjugate crude product (lanes 2 and 3) and G2 conjugate crude product (lanes 6 and 7) were run along with unmodified DNA (lanes 4 and 5), and unmodified DNA mixed with acetate-terminated G3 PAMAM dendrimers (lanes 1 and 8). Acetate-terminated dendrimers were used to detect potential electrostatic complex formation between the protonated tertiary amines of the dendrimer and the polyanionic DNA backbone. The G3 dendrimer is comparable in size to the G4 dendron used for conjugation. The gel shows that a mass shifted band of DNA is present in only the lanes containing the crude DNA-dendron conjugates, with the G2 conjugates having migrated farther than the G4 conjugates as expected from their size.

To further verify that the upper band present in the product lanes is the conjugate, the gel was stained with coomassie blue G-250 protein reagent for 1 hour (Figure S3(right)). Coomassie blue is usually used to stain proteins by complexing an anionic form of the dye with positively charged residues such as arginine and lysine ${ }^{3}$. Since the dendrons have interior tertiary amine groups, the dye complexes with the dendrons and was used for visualizing them on the gel. A definite dendron band below the wells can be seen only in the conjugate lanes that lines up with the EtBr bands.

Upon confirmation of successful DNA/dendron conjugation, the conjugates were excised and purified using the crush and soak method of DNA purification ${ }^{4}$. After purification, the conjugates were run on a $4 \%$ agarose gel as shown in Figure S4 to confirm successful purification. Purified conjugates (lanes 3 and 4) were run along with unmodified ssDNA (lanes 1 and 6) and crude 
product (lanes 2 and 5). The inset of the figure gives an overexposed image emphasizing the purified conjugates and absence of ssDNA bands. Successful purification is indicated by the presence of only one band in the conjugate lane that runs with the conjugate bands in the crude product. Confirmation of successful conjugation of DNA to G4 dendrons allows the conjugates to be isolated and used to study their structure and function when used to construct monolayers.

Figure S3: An agarose gel used to confirm the presence of DNA/dendron conjugates (left) stained with EtBr to visualize DNA and (right) the same gel stained with coomassie to visual dendrimers. Lanes 1 and 8: unmodified ssDNA and acetate-terminated G3 dendrimers mixed without conjugation, Lanes 2 and 3: G4 dendron conjugated to ssDNA, Lanes 4 and 5: unmodified ssDNA, Lanes 6 and 7: G2 dendron conjugated to ssDNA.

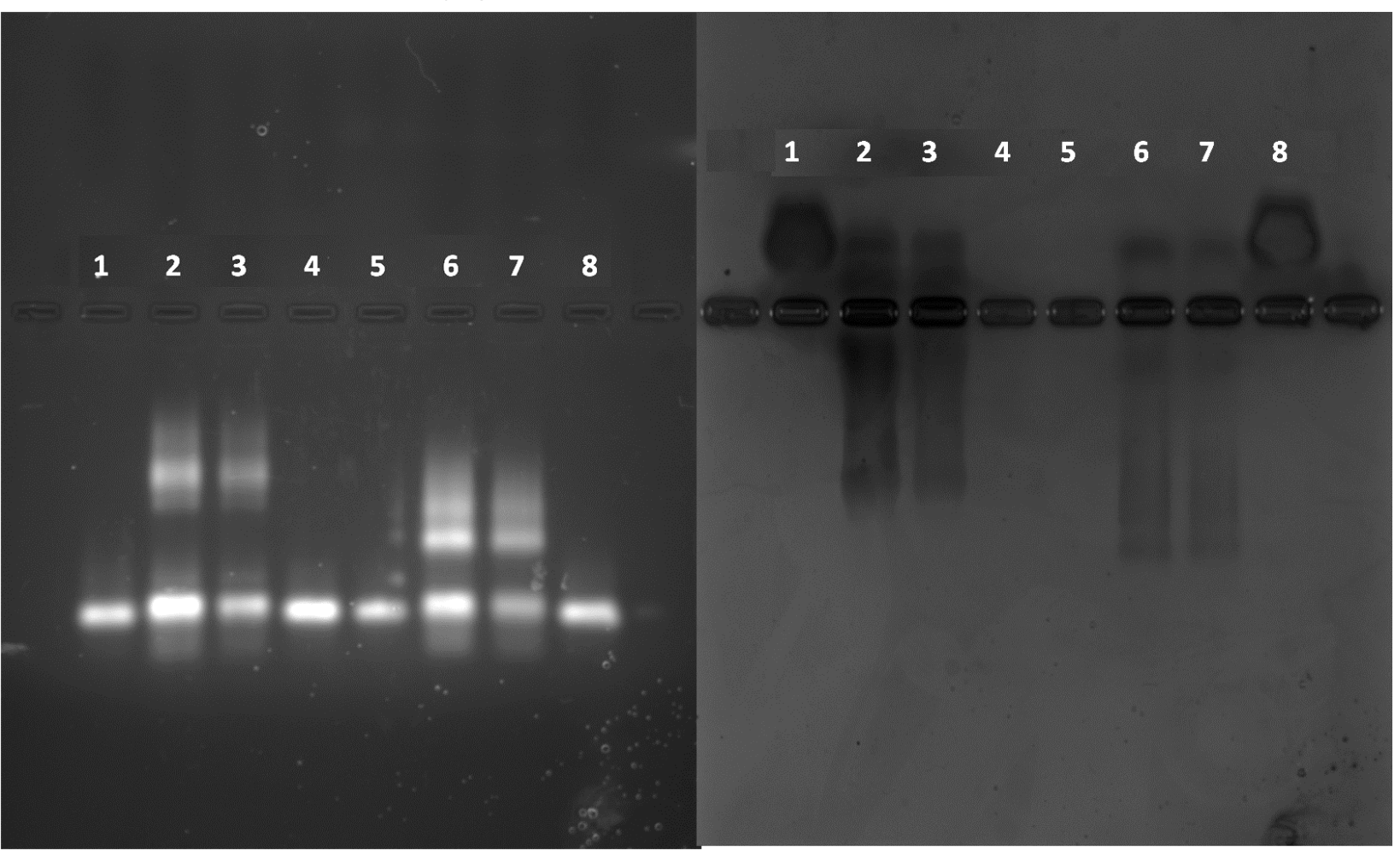




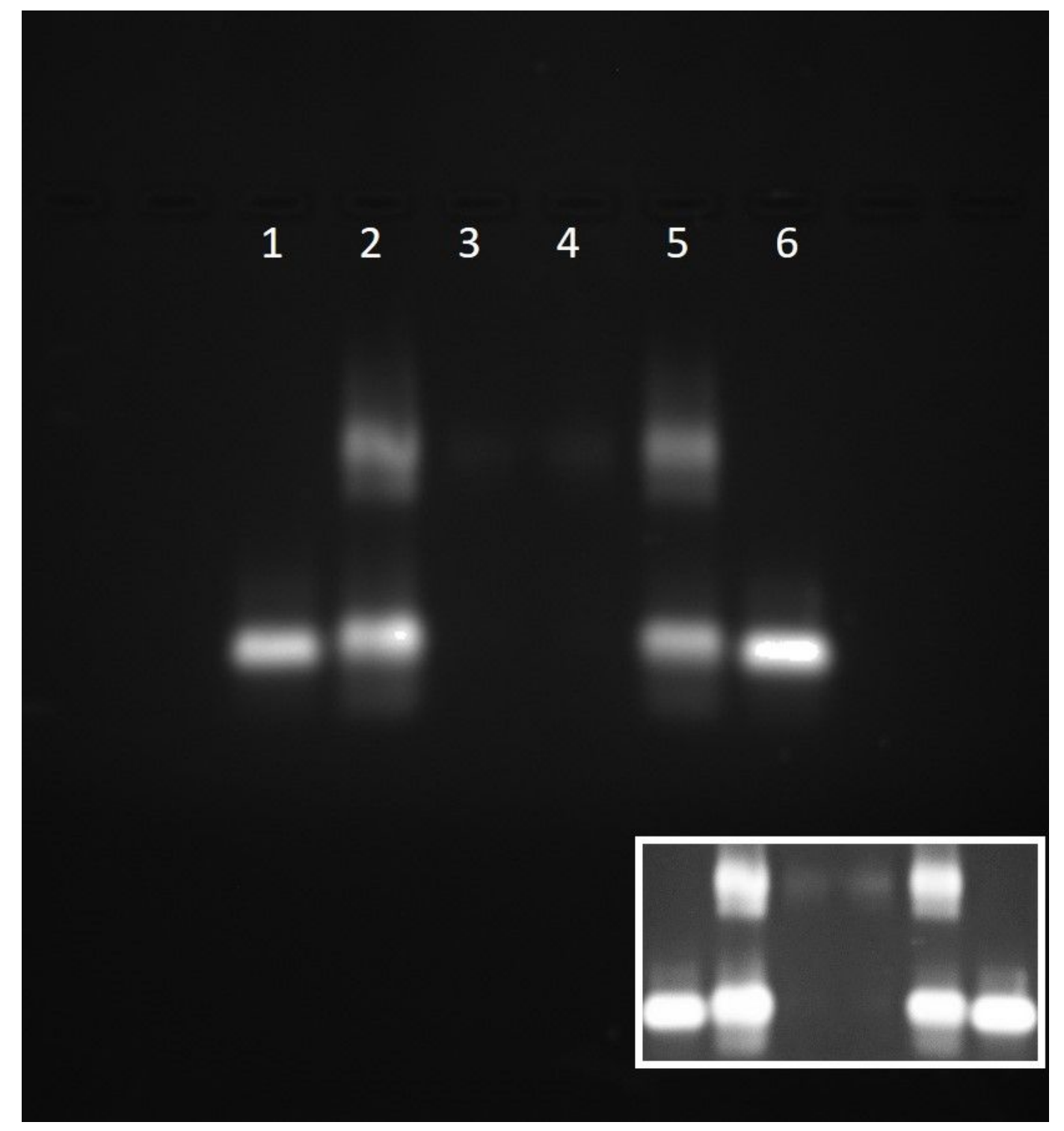

Figure S4: An agarose gel used to confirm the purification of DNA/dendron conjugates. Inset shows an overexposed image zoomed in around the bands. Lanes 1 and 6: unmodified ssDNA, Lanes 2 and 5: G4 dendrons conjugated to ssDNA, Lanes 3 and 4: purified DNA/G4 dendron conjugates.

\section{b. Grazing Angle Infrared Spectra}


Figure S5 shows the IR spectra for the generation 2, 3, 4, and 5 DNA-dendron conjugate monolayers. As noted in the article text, peaks at $1083 \mathrm{~cm}^{-1}$ and $1231 \mathrm{~cm}^{-1}$ are from the symmetric and antisymmetric stretches of the $\mathrm{PO}_{2}^{-}$groups in the DNA backbone, the peaks in the region from 1600 to $1750 \mathrm{~cm}^{-1}$ are from $\mathrm{C}=\mathrm{N}$ and $\mathrm{C}=\mathrm{C}$ stretching modes as well as carbonyl stretching modes from the DNA bases, and the peaks centered around $1675 \mathrm{~cm}^{-1}$ and $1550 \mathrm{~cm}^{-1}$ are the amide 1 and 2 bands, respectively.

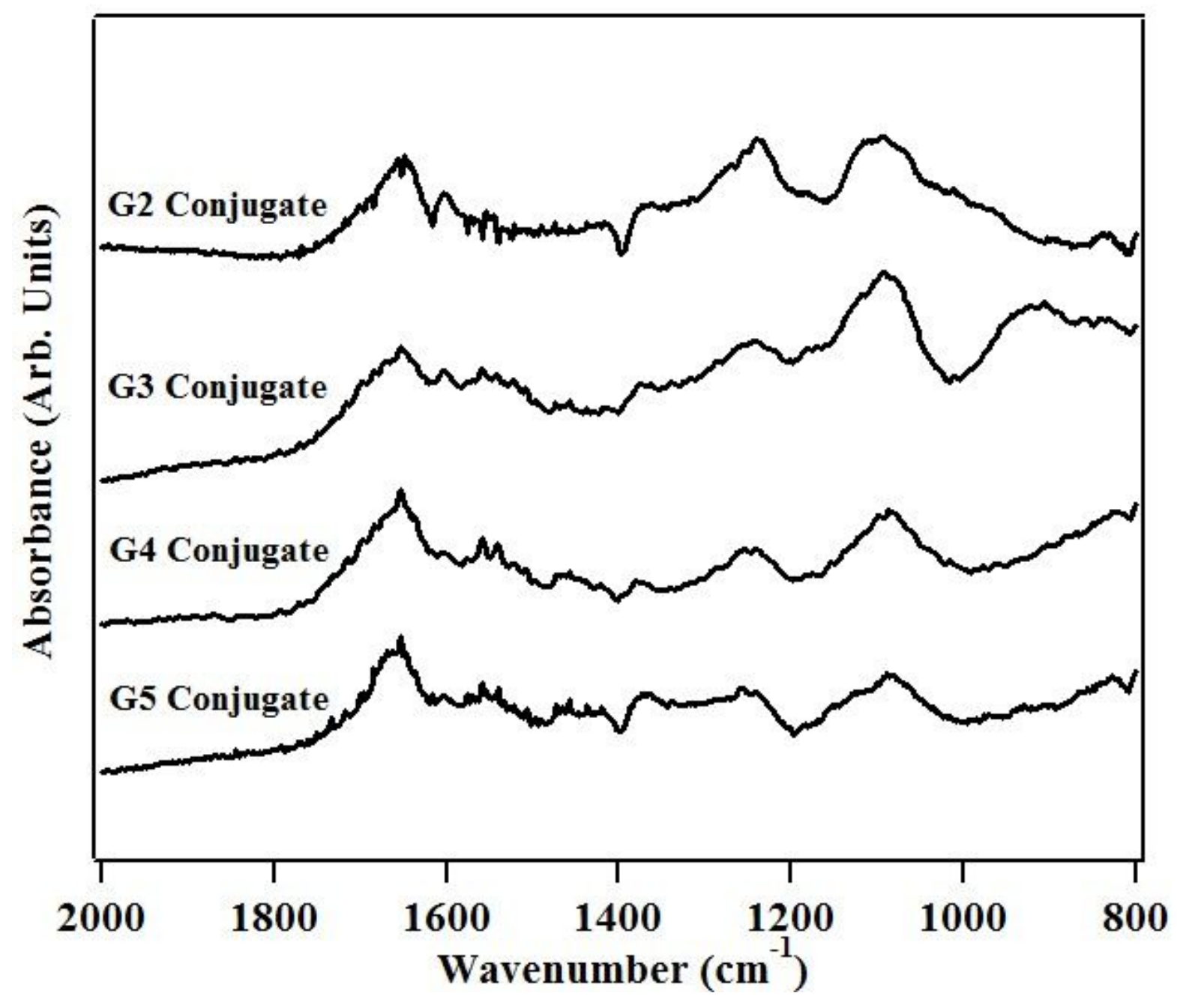


Figure S5: Grazing Angle Infrared Spectra of Generation 2, 3, 4, and 5 DNA-Dendron

Conjugate Monolayers on Gold 


\section{References:}

1. Peterlinz, K. A.; Georgiadis, R. M., Observation of Hybridization and Dehybridization of Thiol-Tethered DNA Using Two-Color Surface Plasmon Resonance Spectroscopy. Journal of the American Chemical Society 1997, 119 (14), 3401-3402. 2. McMeekin, T. L.; Wilensky, M.; Groves, M. L., Refractive Indices of proteins in relation to amino acid composition and specific volume. Biochemical and Biophysical Research Communications 1962, 7(2), 151-156.

3. Georgiou, C. D.; Grintzalis, K.; Zervourdakis, G.; Papapostolou, I., Mechanism of coomassie brilliant blue G-250 binding to protiens: a hydrophobic assay for nanogram quantities of proteins. Analytical Bioanalytical Chemistry 2008, 391 (21), 820.

4. Chen, Z.; Ruffner, D. E., Modified crush-and-soak method for recovering oligodeoxynucleotides from polyacrylamide gel. Biotechniques 1996, 21 (5), 820-822. 